\title{
Edoardo Robino: lo visible y lo invisible
}

\section{Edoardo Robino: the Visible and the Invisible}

\author{
Jean-Claude LÉVÊQUE \\ CSIC-CCHS- Instituto de Filosofía- Madrid
}

Recibido: 01/01/2011

Aceptado: 17/01/2011

\section{Resumen}

En este artículo pretendemos demostrar la originalidad de la obra de Edoardo Robino, artista italiano cuyo pensamiento no se puede separar de su producción artística: escultura y escritura tienen que considerarse en su unidad, como dos aspectos del mismo camino de interpretación de la realidad. En el mismo sentido, en el pensamiento de Robino, naturaleza y cultura no van separadas, sino que representan las dos caras de un proceso en que el hombre está implicado desde el principio. La identidad es también diferencia, y es por lo tanto irrazonable negar la unidad de naturaleza y cultura. Esta unidad en la diferencia ha sido estudiada por el autor a partir del Estructuralismo y de la filosofía del lenguaje contemporánea y ha dado lugar a un procedimiento del pensar que tiene mucho interés para la Teoría estética actual.

Palabras clave: Escritura, unidad, diferencia, Estructuralismo, proceso.

\begin{abstract}
In this paper we will demonstrate the originality of the work of Edoardo Robino, an Italian artist which thought can't be considered without his artistic production: sculpture and writing must be considered as a unity, as two sides of the same path of interpretation of reality. In the same way, in the thought of Robino nature and culture are not separate, but represent the two faces of a process in which
\end{abstract}


man is involved from the start. Identity is also difference, and therefore is not suitable to deny the unity of nature and culture. This unity in difference has been investigated by the author from a structural point of view and forma the contemporary Philosophy of language, producing a procedure that can be interesting for the contemporary Aesthetics.

Keywords: Writing, unity, difference, Structuralism, process.

\section{Sumario}

1. La teoría de lo cómico en Robino

2. Robino y el Estructuralismo

3. La forma, el pensamiento

Robino escultor y Robino estudioso del lenguaje: dos actividades que ocupan dos lugares distintos pero que no cesan de tener relaciones muy bien definidas entre ellos.

Porque en Robino el estudio de los mecanismos del lenguaje, de sus escruturas no tiene una finalidad en sí, sino que tiene que ver con la posición del hombre en relación con la Naturaleza y con la Cultura, con su orientación y con su procedencia.

La formación del autor, que ha de entenderse precisamente en cuanto Bildung, ha sido esencialmente filosófica -en los años transcurridos en Turín, frecuentando las clases de Pietro Chiodi y de Luigi Pareyson. Esta característica, unida a su refinada sensibilidad para la "fenomenología" del lenguaje explica la seguridad absoluta con la cual él se ha movido al interior del Estructuralismo, incluso en sus más intricados desarrollos, teniendo siempre en cuenta la preciosa enseñanza de Saussure, del cual era profundo conocedor. ${ }^{1}$

Al lado del lingüista ginebrino, otro autor que ha sido objeto de un estudio pormenorizado por parte de Robino ha sido J. Lacan (y sobre todo el Lacan de los Escritos). La teoría del signifiant desarrolada por Lacan a partir de la dicotomía saussuriana ha sido constantemente tenida en cuenta por Robino en sus estudios sobre el lenguaje, y esa fuerte presencia se puede notar incluso en el otro ámbito de estudios privilegiado por el autor, el de lo cómico considerado desde un punto de vista lingüístico y antropológico al mismo tiempo.

El Estructuralismo, en suma, interesa a Robino en la medida en que intenta dar cuenta de la estructura profunda del lenguaje humano y, al mismo tiempo da cuen-

\footnotetext{
${ }^{1}$ Un texto muy trabajado por Robino ha sido: Jakobson, R., Saggi di lingüística generale. Milano, Feltrinelli, 1966.
} 
ta de la convivencia de la unidad y de la diferencia en todas las producciones humanas. El concepto de trazo diferencial desarrollado por Jakobson permite a Robino de desarrollar unos análisis muy complejos sobre las relaciones de igualdad y de diferencia que caracterizan a los idiomas, trabajando sobre los juegos de palabras y los dobles sentidos desde un punto de vista fonético, sintáctico y semántico al mismo tiempo.

El juego como tal, en cuanto actividad extremadamente seria y al mismo tiempo despreocupada mueve las preocupaciones intelectuales del autor. Así, según me parece, entiende el juego Robino: más importante que el sujeto que juega es el juego en sí mismo, sea ese lingüístico o de otro tipo, ya que a ese se refiere una serie de reglas implícitas que están aceptadas por los jugadores. En el lenguaje el juego es precisamente lo que hace ver $\mathrm{y}$, al mismo tiempo, esconde las reglas de transformación que presiden a su actuación: las transformaciones del significante implican, evidentemente, las transformaciones al nivel del sentido.

La misma contradicción (entendida propiamente en cuanto contra-dicción) aclara aquello aspectos ocultos del mundo que de otra forma estarían perdidos en la visión cartesiana "clara y distinta", como en la lógica del Círculo de Viena. El lenguaje, para Robino, está siempre estrechamente relacionado con el deseo, con el nomanifiesto. Detrás de la siguiente afirmación, topamos otra vez con Lacan: "es el deseo lo que mueve la escritura."

Deseo y escritura, deseo y lenguaje son los elementos (invisible el uno, visibles los otros) del juego en el cual el hombre está prendido ya desde el principio en su co-pertenecer a la naturaleza.

Las formas lingüísticas están producidas por un principio de transformación que es visible para nosotros sólo en las relaciones de oposición propias de los rasgos diferenciales: podemos suponer su existencia, sin embargo él no se muestra como tal. Como recuerda Lacan en su escrito La signification du Phallus:

C'est ainsi que certains auteurs ont été amenés a considérer la phase phallique comme l'effet d'un refoulement et la fonction qu'y prend l'objet pahhlique comme un symptôme. La difficulté commence quand il s'agit de savoir quel symptôme. Phobie dit l'un, perversión dit l'autre, et parfois le même. Il apparaît dans ce dernier cas que rien ne va plus: non pas qu'il ne se présentent d'intéressantes transmutations d'objet d'une phobie en fétiche, mais précisement si elles sont intéressantes, c'est pour la différence de leur place dans la structure $(\ldots)^{2}$

\footnotetext{
2 Lacan, J., Écrits II. Paris, Seuil, 1971, p.105. Traducción: “ Es así cómo ciertos autores han sido empujados a considerar la fase fálica como el efecto de una represión y la función que ahí tiene el objeto fálico como un síntoma. La dificultad comienza cuando se trata de saber cuál es el síntoma. Unos dice que es fobia, otros que perversión, y a veces las dos. En este último caso, parece que ya no vale nada: no que no se manifiesten ciertas interesantes mutaciones del objeto en fetiche, sino que precisamente, si ella son interesantes, es sólo por causa de su posición en la estructura...".
} 
Es a partir del concepto de diferencia (en la unidad) que la cita del texto lacaniano permite aproximar la complejidad del pensamiento de Robino, que no se reduce nunca a una meditación sobre las consecuencias del método estructuralista sino que supone un revisión de lo reprimido en la tradición filosófica occidental, de esas posiciones que surgieron por poco tiempo o en las reflexiones de pocos autores marginados y luego cayeron en el olvido, sobrepasadas por la violencia de la metafísica.

En el pensamiento de Robino, "el uno es lo otro = el uno y lo otro": la diferencia está incluida en la identidad y la identidad en la diferencia. Se explica de esa forma el interés del autor en la poesía de Hölderlin, centrada sobre todo en los textos de la madurez.

La poesía Der Winter está entre las que más le han interesado a Robino, ya que en ella aparece con toda claridad la relación entre el cambio y el permanecer, entre los signos invisibles del invierno y lo invisible anuncio de la primavera que está por llegar y en todo caso ya comprendida en el principio de la alternancia de las estaciones:

Cuando pálida nieve embellece el campo

$\mathrm{Y}$ alta luz mira la larga llanura

El estío es una seducción lejana;

Mas a menudo se acerca levemente

La primavera mientras que el momento

Se hunde. ${ }^{3}$

Naturaleza y lenguaje no son vistos como realidad separadas, sino que como manifestaciones de un mismo principio de alternancia y de transformación; harmonía y disonancia no son más que las dos caras de una misma realidad que se da justamente en una forma $y$ en la otra, mas que no consiste de dos partes separadas y divergentes.

La búsqueda hölderliniana de la harmonía se encuentra con la reflexión de Robino sobre el lenguaje en cuanto estructurarse de trazos diferenciales y unidad de permanencia y variación. La misma orientación de pensamiento empuja Robino a leer otro filósofo muchas veces malentendido por su presumida oscuridad: Heráclito de Éfeso.

La imagen heraclitea del xunein, de la concatenación de todo lo que acaece coincide con la idea de naturaleza de Robino, naturaleza en la que cada acontecimiento está relacionado con otro y no está separado de los demás.

Como recuerda Giorgio Colli, “Arriba de lo universal visible del fuego está la suprema individuación divina, la cual recoge en una forma inmutable la compleji-

\footnotetext{
3 Hölderlin, F., Le Liriche, trad. di E. Madruzzato. Milano, Adelphi, 1977, p. 857 (tr. al castellano nuestra).
} 
dad de la apariencia y representa el fundamento en que se unen las dos leyes supremas del fenómeno, guerra-paz, en sus actuaciones cualitativas, saciedad-hambre y cuantitativos, noche-día (...). ${ }^{4}$

Así para Robino lo que no se ve es tan importante como lo que se ve y está a la mano y el cambio, la variación se hace comprensible sólo si se consideran al mismo tiempo lo visible y lo invisible.

Esta es entonces la gran intuición a la que el autor será fiel en toda su trayectoria vital e intelectual, hasta que culmine en los Pensamientos de 1986, que constituyen la síntesis de una larga labor sobre el cómico y sobre la relaciones entre naturaleza y cultura. En la forma del aforismo, Robino condensa y refina la percepción de una realidad que es de una forma pero también de otra, y nunca reductible al ser como permanecer, como principio onto-teológico.

De éstos aforismos, me parece oportuno citar el siguiente, como botón de muestra de las reflexiones sobre poesía y lenguaje:

Que la poesía ponga el relación el sonido con el sentido no quiere decir que tenga que ser recitada (leída) par ser entendida, significa que ella re-instaura un antiguo pacto entre el ser y su mundo.

En el lenguaje poético, la palabra "repite" la operación que ha dado lugar al lenguaje, operación natural y artificial a la vez. En ello es posible para el hombre reencontrar aquella harmonía y comunión con la naturaleza -o, por lo menos, una huella de ella- que había sido perdida en el proceso de civilización.

La creatividad del lenguaje es constantemente puesta en relación con el proceso natural de creación y transformación, en el que las cosas se encuentran ser; al mismo tiempo, las misma y, sin embargo, otras; de la misma manera, en el fenómeno de la aliteración que el autor ha estudiado mucho tiempo, son los elementos diferenciales (fonemas) los que hacen que dos términos sea similares mas distintos y que a una variación fónica corresponda una variación de sentido. Lo que divide une, y lo que une, al mismo tiempo, divide. En esto naturaleza y lenguaje pueden encontrarse. ${ }^{5}$

\footnotetext{
4 Colli ,G., la natura ama nascondersi. Milano, Adelphi, 1988, p.208 (tr. nuestra). Del mismo Colli, Robino leyó muy atentamente Filosofia dell'espressione. Milano, Adelphi, 1976 (Hay traducción al castellano, Filosofía de la expresión. Madrid, Siruela, 1996). De este texto verdaderamente muy complejo, podemos sacar una cita muy útil para entender la relación entre necesidad y contingencia en Robino: "La segunda definición de necesario y contingente será metafísica: lo necesario es la categoría que expresa la representación como nexo, en tanto que manifiesta en el logos el aspecto de violencia de las expresiones primeras; lo contingente es la categoría que expresa la representación como nexo, en tanto que manifiesta el aspecto de juego de las expresiones primeras"(Ibid., p.121).

5 Como ha señalado muy bien PaoloVirno, en su ensayo E cosí via, all'infinito. Logica e antropologia. Torino, Bollati Boringhieri, 2010: "El incesante intercambio de papeles entre las dos polaridades -la prevalente se hace subordinada, la subordinda prevalente- muestra en la forma más clásica la uni-
} 


\section{La teoría de lo cómico en Robino}

El largo análisis que Robino dedica a lo cómico puede dividirse en tres partes fundamentales.

a. El estudio del ensayo freudiano El chiste, con una crítica de las teorías freudianas,

b. El análisis de lo cómico en Rabelais;

c. Una larga y compleja investigación sobre los mecanismos de lo cómico a partir de unas tiras y de los chistes más conocidos para encontrar un paradigma común del efecto cómico.

Desde luego, el análisis del texto de Freud no puede separase del complejo de las investigaciones del autor, sino que tiene que ser insertado en el corazón mismo de su reflexión sobre el lenguaje y la naturaleza, aquí llevada a un nivel máximo de rigor y sistematicidad. 6

a. Robino, en el análisis del ensayo freudiano, demuestra ser un lector sensible y crítico, atento a todos los matices del texto: su interés se centra en el estudio del mecanismo complejo presente en el efecto cómico: lo cómico es un efecto lingüístico que tiene que ver con el inconsciente y con la autocensura que el hombre impone a una parte de su material psíquico.

Dos son los fenómenos que Robino analiza en el detalle: el fenómeno de condensación y el de ahorro. Así se expresa el mismo Freud sobre la cuestión.

Un ahorro localizado como el que acabamos de considerar no dejará nunca de proporcionarnos un momentáneo placer, pero no producirá jamás una duradera economìa mientras lo economizado pueda ser empleado en otro lugar.

Sólo cuando este distinto empleo puede ser evitado se transforma de nuevo el ahorro especial en una minoración general del gasto psíquico. Aparece, pues, ahora que hemos profundizado más en nuestro conocimiento de los procesos psíquicos, un nuevo factor: la minoración en lugar de la economía. Vemos claramente que el primero produce una sensación de placer mucho más importante. ${ }^{7}$

dad y la distinción entre biología y cultura; mejor aún, muestra la imposibilidad de separa su unidad y distinción". (p.43, tr. nuestra).

6 En la introducción a su breve ensayo Motto di spirito e azione innovatiiva (Torino, Bollati Boringhieri, 2006), P. Virno afirma: “ la segunda hipótesis subordinada suena así: la forma lógica del chiste consiste en una falacia argumentativa, o sea en una inferencia indebida o en el uso incorrecto de una ambigüedad semántica: por ejemplo: atribuir al sujeto gramatical todas las propiedades que pertenecen a su predicado, intercambiar el todo por la parte o la parte por el todo, instituir una relación simétrica de antecedente a consiguiente, tratar a una expresión metalingüística como si fuera un lenguaje-objeto" (p.VI- traducción nuestra).

7 Freud, S., El chiste y su relación con lo inconsciente. Madrid, Alianza ed., 2000, p. 157. 
Ahora bien, si a Freud lo que le interesa en primer lugar es la relación del chiste con el inconsciente, y desde un punto de vista que podríamos considerar epistemológico y fundamental, Robino en cambio lee el ahorro primero desde el punto de vista del lenguaje, y luego le relaciona con la expresión/ represión de un deseo.

Por otra parte, cuando analiza el mecanismo de los chistes que se cuentan en un ambiente familiar y de amistad, nota que

Apparentemente si ride a vuoto, nella misura in cui manca la costruzione di un effetto tale per cui si rida. Questo succede in genere in situazione, "come di amici e sodali", e mai tra estranei.

Gli amici infatti condividono degli effetti(tali da ridere) che i non amici ignorano, non è che per essere amici ridevano anche quando non c'era nulla da ridere.

Se l'essere in intimità tocca l'intimità di ciascuno e se l'intimità propri va di pari passo con la propria perdita (vedi Lacan, la fase dello specchio), col rifiuto, la negazione, non è strano che gli intimi ridano avendo a che fare nella loro intimità con ciò che non vogliono dire ma che è da dire (cart. 4, 1977, p.1).

Donde el análisis freudiano está completado a través del análisis lacaniano del lenguaje y del inconsciente, a través del tema de la constitución de la identidad.

Freud, en última parte de su ensayo, considera que lo cómico tiene que ver con "la representación mediante lo contrario"8; Robino empieza justamente por esta observación de Freud a construir su propia teoría de lo cómico, demostrando como tenga que ver al mismo tiempo con el funcionamiento del lenguaje y con lo del pensamiento. A partir de un caso peculiar, Robino pretende reconstruir el funcionamiento de lo cómico en general, manteniendo una tensión hacia la unidad de los contrarios que caracteriza, como hemos visto, toda su investigación.

Un ejemplo sacado de Freud nos puede ayudar a entender mejor el procedimiento de Robino:

Wippchen se sirve, sobre todo, de las fusiones, de la modificación de lugares comunes y conocidas citas literarias y de la sustitución de triviales elementos de las mismas por expresiones más altisonantes, técnicas que no se paratan mucho de las del chiste.

Un ejemplo de fusión: No es más que una seca columba que testimonia de antiguas grandezas", frase resultante de la condensación de otras dos, "un seco árbol" y una "columba que...", conocidísimos ambas como lugares comunes de la literatura pedestre. 9

La fusión (mantenemos aquí ese término por reverencia a Freud, bien que este no aclare del todo la dirección de la investigación de Robino), permite la coexistencia de dos significados posibles, garantizando aquella ambigüedad semántica que solo puede dar lugar a lo cómico.

\footnotetext{
8 Ibidem, p. 205.

9 Ibidem, p. 218.
} 
Freud analiza otro aspecto del chiste que ha llamado la atención de Robino: el desperdicio. Freud recuerda el hecho de que

El placer cómico y el efecto en que el mismo se manifiesta -o sea la risa- no pueden surgir sino cuando tal diferencia deviene inútil y, por tanto, susceptible de descarga.

Cuando, por el contrario, recibe inmediatamente después de su aparición cualquier otro empleo no experimentamos ningun efecto de placer, sino a lo más, una fugitiva sensación placentera exenta de todo carácter cómico. ${ }^{10}$

Robino reconoce la pertinencia de las observaciones de Freud sobre el desperdicio subrayando el hecho de que, en este balance energético, el sujeto esté implicado en lo que le es más propio: en el chiste, el sujeto pone en juego sí mismo y aquella parte de sí que menos querría desvelar, a través de una implicación que se echa a perder, sin un interés inmediato.

Para Robino pensamiento y lenguaje funcionan "al vacío" sobre todo en lo cómico y por eso este es apto de forma peculiar a desvelar nuestro funcionamiento en cuanto seres humanos.

Nosotros podemos añadir que existe una especie de double bind entre el pensamiento y el lenguaje que no actúa nunca en un único sentido, sino que siempre presupone el principio de reciprocidad: en la co-presencia de dos significados distintos en el chiste y en su necesidad y gratuidad se encuentra la raíz de la producción de sentido por parte del hombre, producción que está siempre caracterizada por la complejidad.

\section{a. Lo cómico en Rabelais. Una investigación estructural}

La obra de Rabelais ha fascinado enormemente a Robino precisamente por la peculiaridad y la profundidad de los mecanismos de lo cómico utilizados por el humanista francés.

El autor aquí ha analizado los cinco libros de Rabelais, realizando un trabajo de cuño estructural, teniendo sin embargo en cuenta que Robino no se atiene nunca a la letra del Estructuralismo sino que ese constituye más bien un instrumento para aclarar su propio proyecto filosófico en su conjunto.

Me parece oportuno alegar un ejemplo concreto para que quede más claro su método y las finalidades que se había propuesto:

(cit. Da Rabelais, Gargantua, cap. XXVII, p.109, ed. Classiques Garnier) ${ }^{11}$

\footnotetext{
10 Ibidem, p. 222.

11 Traducción: "Ejemplos de cómo un objeto pueda volverse equívoco, a través de una transformación. Ciertamente decisiva, pero tal que parezca siempre el mismo objeto para que las dos funciones coe-
} 
...les tabourineurs avaeint defence leurs tabourins d'un couté pour les emplir de raisins, les trompettes avoient éstés chargez de moussines...

Esempi di come un oggetto possa farsi equivoco, attraverso una trasformazione, decisive sì, ma tale da sembrare sempre los stesso oggetto in modo che due funzioni coesistano (in questo caso la cavità sonora e la cavità per contenere), l'una come affermazione, l'altra come negazione (a scelta, a seconda di quello che si vuole ottenere) (Cart. 18, p. 40).

El equívoco es la forma primaria de lo cómico en Rabelais: los términos empleados están sometidos a una oscilación semántica de manera que su sentido no esté nunca decidido una vez para siempre, sino que esté siempre por decidir. La transformación del término 8 y de la estructura lingüística en la que está insertado adviene de manera que los dos significados coexistan y estén relacionados el uno con lo otro.

Sobre este concepto de transformación es interesante remitir a otro texto de Robino, donde se aclara mejor la necesaria coexistencia de transformación y persistencia en el efecto cómico (en cuanto effet de pensée y efecto lingüístico al mismo tiempo):

Rabelais, Cap. XXXII, p.124

D'une chose, ...vous veux-je advertir..

Che per mancanza di cibo, in caso di assedio, si farà, e si farà a tutti, strappare i denti, lasciandone solo tre. Perché meno denti si ha, meno si mangia.

Alcuni effetti di riso:

Gli uomini che mangiano, si procurano (uccisione e trasporto) il cibo coi Denti (fiere), niente Denti, niente cibo.

Regolarità dei tre denti, la regolarità forse è massima all'origine, quando uno (salve eccezioni) li ha tutti e trentadue. Da quel momento in poi subentra l'infinita varietà del deterioramento, del consumo...Quindi che tutti abbiano tre denti, è come dire la infinita varietà dell'uniformità... (ibid, p.44) $)^{12}$

En este caso se nota cómo el cambio presenta al mismo tiempo regularidad y variedad: todo es distinto, pero al mismo tiempo todo es como antes (cuando todos tenían sus dientes).

xistan (en este caso la cavidad sonora y la cavidad como recipiente), la primera como afirmación, la segunda como negación (según la elección, lo que se quiera obtener".

12 Traducción: "Que, en caso de falta de comida, vamos a arrancar, y a todos, los dientes, dejando a cada uno sólo tres, ya que menos dientes se tiene, menos se come.

Algunos efectos de la risa:

Los hombres que comen, se procuran (matanza y transporte) la comida con los dientes (fieras): si no hay dientes, no hay comida.

Regularidad de los tres dientes, la regularidad de los dientes quizás sea máxima en el principio, cuando uno (menos algunas excepciones) los tiene todos. A partir de aquel momento se impone la infinita variedad del deteriorarse, de la consumación... Entonces el que todos tengan tres dientes quiere decir algo como la infinita variedad en la uniformidad..." 
Es como si, presente pero invisible junto a los trazos pertinentes, hubiera un "tercer trazo" -y así lo nombramos aunque no sea un verdadero tercio- el cual une los demás y les proporciona sentido.

Sin unidad, no hay diferencia y sin diferencia, no hay unidad. El efecto de lo cómico manifiesta significativamente la dirección del deseo que queda en todo caso invisible detrás de los efectos estructurales.

La investigación de Robino por eso es una investigación sobre una forma: la del cómico como forma de lenguaje y como lenguaje de la forma. En el Gargantúa et Pantagruel el autor encuentra el desarrollarse y el perfeccionarse de una forma que es, al mismo tiempo un modelo del funcionamiento de la mente:

...defendre... ofender... que peu de chose me retient que je n'entre en l'oppinion du bon Héraclitus, affermant guerre etre de tous biens père... (p. 397)

Quando c'era la pace, litigavano tutti, quando c'era la guerra erano tutti in accordo. Ovvero in pace. Quando c'è pace c'è guerra e viceversa (Ibid., p. 91).13

Aquí resulta claro que lo que importa es la forma de lo que se dice, ya que sólo así es posible entender su sentido. 14

El cómico en Rabelais es un hecho de forma, que hace explícito un mecanismo de pensamiento: una condensación que permite un ahorro energético.

\section{b. La forma de lo cómico}

A través del análisis de unas tiras Robino intenta aislar el mecanismo de lo cómico en su forma más pura, después del largo trabajo hecho sobre Freud y Rabelais.

El autor intenta mostrar que lo que genera el efecto cómico es precisamente la misma forma, que induce la ambigüedad. En el chiste, la ambigüedad del texto impide, por ejemplo, distinguir entre el sentido literal y el metafórico, lo que genera la risa.

\footnotetext{
13 Traducción. "Cuando había paz, se peleaban todos entre ellos, cuando estaban en guerra, estaban todos de acuerdo. O sea en paz. Cuando hay paz hay guerra, y viceversa."

14 Quisiera aquí citar una breve nota de un lingüista hoy olvidado, Antonino Pagliaro, que Robino había leído con mucho interés: ...el hombre, en cuanto piensa y habla (lógos es evidentemente el pensamiento en la forma discursiva), revela la ley que mueve todo lo real del cual él participa (...) El proceso de la conexión (...) por lo que se opera la síntesis de los opuestos tiene claramente su modelo en el proceso sintáctico". (Pagliaro, A., Saggi, Roma, 1953, p.133 e 154.

La conexión entre pensamiento y lenguaje es aceptada también por Robino, que radicaliza la posición de Pagliaro en un sentido formal.
} 
(...) nel caso del ridere, la differenza non è un'esclusione, ma un'implicazione. Non si tratta di due cose (una differente dall'altra), ma come se Fosse una sola da due, così: $A=(a b) ; b=(a b)$

$\mathrm{a} / \mathrm{b} \quad \mathrm{a} / \mathrm{b}$

con le conseguenze che ne vengono e il risparmio (Cart. 4, p.19).

los dos términos no se excluyen, sino que están implicados el uno en lo otro; no tenemos oposición, sino que co-presencia, unidad.

Alteridad/Identidad; lo que es otro, es al mismo tiempo idéntico. En el análisis de Robino, la forma prevalece sobre el contenido y lo determina (determina sus efectos); lo importante es guardar a la forma, aunque se expresen cosas distintas, ya que es ella la que confiere al chiste su sentido.

Non si ride della distrazione quale causa dello scambio, l'effetto è di forma, e quindi di sostanza. La distrazione è sostanza pura, senza forma, cioè niente (Ibid., p. 25).15

Otra nota de Robino contribuye a aclarar esta identidad en la diferencia en cuanto efecto de la forma:

L'annullamento della differenza non significa passare a parlarne in termini di uguaglianza (come a dire: sitratta dello stesso sguardo, uguale a se stesso), ma significa, nei casi di cui ci occupiamo, che un'identità (da identico) è falsa, che essa nasconde qualcosa che le è differente senza parlare d'altro (Ibid., p. 27).16

O sea: la identidad visible esconde una diferencia invisible y que está implicada en ella. La estructura fundamental de lo cómico consiste entonces en expresar una cosa así como si fuera otra, manteniéndose en la ambigüedad de la co-presencia del sentido literal y de lo metafórico (o simplemente de los dos sentidos posibles).

A través de un efecto de estructura, el mecanismo de lo cómico se revela, pero de forma indirecta (es algo que hay que entender, no algo dado); la ambigüedad de la información es una función de la condesación y de la concentración estructural del enunciado. ${ }^{17}$

15 Traducción: "No se ríe de la distracción en cuanto causa del intercambio, el efecto es formal, y entonces sustancial. La distracción es pura sustancia, sin forma, o sea nada".

16 Traducción: " La anulación de la diferencia no significa pasar a hablar en términos de igualdad (es decir: se trata de la misma mirada, igual a sí misma), sino que significa, en los casos que tratamos, que una identidad (de "idéntico") es falsa, que ella esconde algo distinto sin hablar de otra cosa".

17 Sobre el hecho de que el pensamiento tenga una semántica combinatoria, se puede ver de Fodor, J.A., La modularidad de la mente. Madrid, Morata, 1986. 
Lo cómico, en cuanto hecho de lenguaje y en cuanto hecho de pensamiento está insertado en una visión "holística" de la mente del hombre: por eso no se presenta sino en la combinatoria lingüísticas y en su poner diferencias (en la unidad) sin generar exclusiones.

\section{Robino y el Estructuralismo}

El encuentro del autor con el Estructuralismo se produjo durante sus estudios universitarios y se inserta con toda claridad en el ámbito de su investigación sobre la relación entre Natura y Cultura.

La lectura del Curso de lingüistica general de Saussure y también de otros clásicos de la lingüística de siglo veinte (Jakobson, Benveniste, Hjemslev...) contribuyó en larga medida al desarrollo de sus reflexiones sobre el lenguaje.

Para aclarar la relación del autor con el Estructuralismo, es necesario citar un pasaje del importante ensayo de Milner, Le périple structural (2002), en que el lingüista francés observa, a propósito de Lacan y de su versión del Estructuralismo,

Una conseguenza dello strutturalismo forte è che il predicato "essere strutturato come un linguaggio" rischia incessantemente di diventare tautologico: un linguaggio non ha niente di più che le proprietà della struttura, ma siccome non vi sono proprietà se non attraverso una struttura, la circolarità salta agli occhi.

Questa può essere evitata solo ammettendo ciò che si può chiamare la congiuntura iperstrutturalista: "la struttura qualsiasi ha proprietà non qualsiasi". ${ }^{18}$

El hiperestructuralismo de Lacan es "un Estructuralismo preso de la letra", en el que Milner observa una obsesión por la cientificidad que puede revelarse demasiado restrictiva.

Pero, si consideramos, más allá de las observaciones de Milner, el interés de Robino por Lacan, veremos que es precisamente ese el punto que le interesa a nuestro autor: ese rasgo suplementario, ese punto de posible novedad que hace que la estructura pueda tener propiedades peculiares: Robino lo encuentra en los Escritos de Lacan y también en Jakobson.

Esta observación puede ser aclarada citando otro trozo de Milner, relativo al segundo Lacan:

\footnotetext{
18 Milner J.-Cl.,, Il periplo strutturale. Milano, Mimesis,2008, p.120 . Traducción: “ Una consecuencia del estructuralismo fuerte es que el predicado " ser estructurado como un lenguaje" tiene el riesgo incesante de devenir tautológico: un lenguaje no tiene más que las propiedades de la estructura, pero, ya que no hay propiedades sino a través de una estructura, la circularidad es evidente. Esta se puede evitar sólo admitiendo lo que puede llamar la conjunctura hyperestructuralista: " la estructura cualquiera tiene propiedades no-cualquiera".
} 
... Non vi è alcuna differenza gerarchica tra elemento e sistema; ogni riferimento all'insieme offusca l'evidenza: nella catena stessa i significanti e la catena non sono altro che la stessa azione pura che si distribuisce tra significanti e catena.

Ogni significante si proietta in quella linea che è la catena (linearità del significante), la catena come linea si proietta in quel punto che è il significante qualsiasi. ${ }^{19}$

A través del Estructuralismo, el autor ha podido precisar la relación entre el hombre y la naturaleza, aclarando además la posición del hombre en esta relación. Siguiendo a Lacan, podríamos hablar de una relación extima, compuesta por una diferencia constitutiva (en el lenguaje), pero también de una unidad constitutiva (el hombre está ya siempre en la naturaleza).

Como subraya F. Frommer,

De là écoulera également le fait-notamment dans l'anthropologie structurale-que le "règne de la culture est le règne de l'échange", de la relation pensée, édifiée, "structurée" entre des différences".20

Los estudios de fonética de Jakobson (1966), además, han por cierto abierto nuevas perspectivas al autor, sobre todo en lo que se refiere a la posibilidad de pensar la unidad en la diferencia.

El insistir de Jakobson en los rasgos diferenciales que definen la contigüidad y las diferencias entre los fonemas han abierto para Robino el camino hacia su propia filosofía.

Como ha resumido con toda claridad Milner, la idea fuerte del Estructuralismo es la que pueda existir "una necesidad thései homóloga a la de la naturaleza": y la misma idea, como hemos visto, se encuentra en los textos de Robino.

\section{La forma, el pensamiento}

a. En Robino la escultura y el pensamiento se encuentran en la cuestión de la forma.

19 Ibidem, p.133 Traducción: “... no hay ninguna diferencia jerárquica entre elemento y sistema: cada referencia al conjunto borra la evidencia: en la cadena misma los significantes y la cadena no son otra cosa que la misma acción en su pureza que se distribuye entre los significantes y la cadena.

Cada significante se proyecta en aquella línea que es la cadena (linearidad del significante) y la cadena como línea se proyecta en aquel punto que es el significante cualquiera."

20 Frommer F., " Comment la France est redevenue le pays le plus anti-intellectuel et le plus anti-politique du monde..." en Mouvements, n.24, nov-déc. 2002, pp.104-112, aquí 108. Traducción. “De allí seguirá el hecho de que-sobre todo en la antropología estructural-“ el reinado de la cultura sea el reinado del intercambio", de la relación pensada, edificada, "estructurada" entre las diferencias." 
La forma del pensamiento y las formas del arte, aunque en su diferencia, son formas de expresión de un ser-lo-mismo: lenguaje y técnicas artísticas se acercan precisamente en su ser procesos, modos de hacer "que inventan su propio hacer mientras que hacen". 21

Escultura y lenguaje son considerados en una relación conjuntiva que no elimina sin embargo las diferencias; su creatividad es de hecho fundamentalmente distinta. Y por eso la una no puede pasarse sin lo otro: son como los dos lados de la misma realidad que se adhieren perfectamente mas que quedan distintos.

En las esculturas de Robino el signo, la escritura (la cual sigue una disposición muy peculiar para producir el sentido) acompañan siempre las formas que surgen del material.

Por un lado el signo, en la estructuración muy peculiar que el autor le impone, tiende a romper la dimensión de la escultura como un todo-un mundo-en sí y para sí, cerrado y que sugiera la inagotabilidad de las transformaciones; del otro la escultura, en cuanto ocupa una espacio determinado y afirma la razones de la materia, de-limita, de alguna forma, el vació de la "página blanca" en que suele inscribirse la escritura. Como afirma Robino,

Scultura: l'indescrivibile. Scrittura: il descrivibile. La scrittura senza scultura diventa interminabile, la scultura senza scrittura diventa "terminata". (Cart. 11, 1984)22

Si la escultura es un dejar-ser a las formas, en una relación con el material que no consiste nunca en una imposición, sino que más bien sigue los "llenos" y los "vacíos" del bloque donde se ejerce la labor del escultor, entonces es precisamente el vacío que permite entrever a las posibilidades formales que se ofrecen al artista y a su mano. Como recordaba Pareyson,

El artista no inventa sólo la obra, sino también su ley: por eso se encuentra en la situación rara de obedecer a la misma obra que está haciendo (...). Es esta, en efecto, la estructura de la normatividad: formar significa hacer, inventando al mismo tiempo el modo de hacer; lo que explica que la actividad artística sea, al mismo tiempo, libertad y necesidad, labor del artista y voluntad de la obra, aventura y orientación; en una palabra, intento y organización. 23

Yo diría que la obra de Robino se inserta precisamente en esta co-presencia de deseo y necesidad, a través de la importancia reconocida de la relación entre naturaleza y artificio.

21 Pareyson, L. Estetica. Milano, Bompiani, 1974, p. 120 (tr. nuestra).

22 Traducción: "La escultura: lo indescriptible. La escritura: lo descriptible. La escritura sin escultura se hace interminable, la escultura sin escritura se hace " terminada".

23 Pareyson, L., Conversazioni di Estetica. Milano, Mursia, 1966, p. 22 (tr. nuestra). Hay una traducción al castellano, Conversaciones de Estética. Madrid, A. Machado, 1988. 
En la labor de la forma, el escultor se inserta en una procesualidad de la que naturaleza y pensamiento-lenguaje representan los dos aspectos, reunidos en la identidad y, al mismo tiempo, distintos: la escritura en los márgenes de la obra reenvía a un sentido posible, mas, de hecho, a lo inalcanzable.

También en este caso ella atraviesa el espacio de la obra que deviene in-terminable. Lo artificial y lo natural se encuentran conjuntos en un efecto de estructura y la obra se vuelve en un camino de sentido, siempre entendido en cuanto como un "proceder-hacia..."24

El "procedimiento" de Robino recuerda, por otra parte, aquello utilizado por R.Roussel en la composición de sus novelas (de Impressions d'Afrique a Locus solus). ${ }^{25}$

Se trata también en este caso de acercar dos términos que mantienen entre ellos una relación paronomástica (o aliterativa) y tienen sin embargo dos significados distintos. El efecto que se obtiene es lo de originar un tercer sentido, que deriva de la relación dialéctica entre los dos términos y a la que es necesario volver a través de una reconstrucción, de un trabajo de explicitación.

La inscripción lingüística reenvía entonces a un procedimiento sobre el cual merece la pena demorarnos para entender en su complejidad las relaciones entre el pensamiento y la obra artística del autor.

\section{b. Pensamiento y forma}

Si intentamos acercarnos a la última parte de la producción filosófica de Robino, veremos que está caracterizada en particular por una amplia colección de Pensamientos (1986) en los que ha querido dar un arreglo formal a su reflexión sobre el lenguaje. Lo que constituye la cifra de este extremo trabajo es la búsqueda de un efecto-de-forma: el autor, a través de aforismos, quiere mostrar el mecanismo de funcionamiento del pensamiento a partir del presupuesto de que pensamiento y lenguaje no se pueden separar, sino que presentan los mismos mecanismos de funcionamiento. Esos modos se pueden entender mejor a partir de la obra misma del autor: consideramos, pues, el pensamiento 503:

La differenza tra noi e le altre forme di vita è che solo a noi interessa. ${ }^{26}$

En este caso la escritura está inspirada por el criterio de la economicidad máxima: esto provoca el acto de atención y el placer de la comprensión a través de la

\footnotetext{
${ }^{24}$ Por eso Robino busca hacer ver los dos momentos complementarios del "mientras" y de lo "interno a...", en la escultura y en el lenguaje.

25 Roussel, R., Comment j'ai écrit certains de mes livres. Paris, Gallimard "L'imaginaire”, 1995.

26 Traducción: "la difrencia entre nosotros y las otras forma de vida consiste en que sólo a nosotros nos interesa".
} 
reconstrucción (la diferencia está dada por el interés para la diferencia, que es propio únicamente de los humanos.).

Sin embargo, este efecto de estructura se ve aún mejor en el pensamiento 305:

Ci sono di quelli che vivono nel loro tempo e di quelli che vivono nel tempo degli altri. 27

Aquí se pueden distinguir dos parejas de significados: $\mathrm{a}=$ su tiempo: contemporaneidad; a' = su tiempo: tiempo de que disponen; $b=$ tiempo de los demás: tiempo otro/ no contemporaneidad; b' = tiempo de que los demás disponen; estos están en una relación dialéctica y para ser entendidos remiten a un tercero no explicitado (que reúne los dos sentidos del enunciado en una síntesis dialéctica, mas conservando su diferencia).

El sentido en su conjunto se encuentra entonces en la forma que une en una relación dialéctica los dos sentidos posibles. En estos breves aforismas emerge por lo tanto con fuerza la necesidad de sacar a la luz la coincidencia entre la forma del pensar y la forma del lenguaje: así como funciona el pensamiento, del mismo modo funciona el lenguaje: sus estructuras, en el juego, coinciden.

Esa estructura de pensamiento me hace pensar en la forma de argumentar propia del primer Hegel (hasta la Fenomenología del Espíritu): así la define P.J. Labarrière, uno de los máximos intérpretes hegelianos:

L 'Unité plurielle (...) tient dans une esquisse dont l'ambition est de decrier, en matière de survol, le movement que de l'immédiat a l'immédiat l'ouvre la totalité du procès de l'intelligence, dans sa double tâche de déchiffrement et de construction, de prise en charge et de transgression; dans ce mouvement bi-directionnel en quoi consiste la culture-à la fois lecture et facture des choses et des mots. ${ }^{28}$

Para Labarrière el pensamiento de Hegel tiene valor en cuanto es pensamiento de lo Otro y de la no-reconciliación, en antítesis con los que lo ven como un sistema cerrado y un pensamiento de la totalidad. Precisamente en la salida de sí mismos y en el reconocimiento de sí a través del ser-para-otro se desarrolla el proceso del adquirir la auto-conciencia considerado dialécticamente.

\footnotetext{
27 Traducción “ Hay los que viven en su tiempo y los que viven en el tiempo de los demás”.

28 Labarrière, P.-J., " D'un style de la pensée: le statut de la figure», en Revue philosophique de Louvain, n.1, (février 1994), pp.15-31, aquí p.23. Traducción: “ La Unidad plural (...) está en un borrador cuya ambición es describir, muy en general, el movimiento que de lo inmediato abarca a la totalidad del proceso de la inteligencia, en su doble tarea de desciframiento y de construcción, de asunción y de transgresión, en breve, en ese movimiento bi-direccional en que consiste la cultura -a la vez lectura y factura de las cosas y de las palabras".
} 
Y con lo Hegel de la Fenomenología había dialogado también Robino, en lo años cerca de los años ochenta del siglo pasado y ayudándose por el comentario de J.Hyppolite.

Ahora tendría que resultar claro que el interés del autor para Hegel no fue casual, sino que Robino se sentía atraído por el filósofo alemán justo por causa de su labor entorno a la forma del pensar.

Sin embargo, hay otro autor importante para Robino en su meditación sobre el pensar: se trata del filósofo americano Ch.S. Peirce.

Una frase de Peirce, sacada del Ms. 439 de 1898 nos puede ayudar a entender las analogías entre su pensamiento y lo de Robino:

¿Qué es la realidad? A lo mejor, no hay nada que se pueda definir así. Como he dicho muchas veces con insistencia, ella no es sino una reintroducción, una hipótesis operativa que ponemos en prueba, nuestra única esperanza, no esperada y miserable, de conocer algo.

Bueno, por otra parte, podría ser -y parecería poco prudente esperar algo más- que la hipótesis que se refiere a la realidad, bien que proporcione respuestas bastantes eficaces, no corresponda exactamente a lo que existe. Mas si alguna realidad existe, entonces, en cuanto existe, consiste en eso: en que en el ser de las cosas hay algo que corresponde al proceso del pensamiento, que el mundo vive, se mueve y tiene su mismo ser en una lógica de los acontecimientos. ${ }^{29}$

Esta correspondencia entre la lógica de la naturaleza y la lógica del pensamiento sustancia toda la reflexión de Robino, y sobre todo la relativa al lenguaje.

Además, la forma triádica de la lógica de Peirce nos proporciona un esquema muy útil a la hora de entender la forma de pensar del autor. ${ }^{30}$

El concepto de relación y la de mediación se acercan a ese tercero que hemos individuado como mediador implícito entre dos sentidos posibles en Robino y que, de alguna manera entra en toda su obra.

La forma del pensamiento, las formas del lenguaje y las formas del arte tienen entre ellas una relación complementaria y dialéctica al mismo tiempo, que explica la complejidad de lo real en cuanto co-presencia de lo visible y de lo invisible en un proceso sin fin.

\footnotetext{
29 Peirce, Ch. S., Categorie, a cura di R. Fabbrichesi Leo. Roma-Bari, Laterza, 1993, pp. 68-69 (traducimos aquí de la edición italiana, porque nos parece más cercana del original).

30 De Peirce se pueden consultar, por ejemplo, en castellano, Escritos lógicos. Madrid, Alianza, 1988.
} 